\title{
Investigating the Effect of Quadruple Therapy with Saccharomyces Boulardii or Lactobacillus Reuteri Strain (DSMZ 17648) Supplements on Eradication of Helicobacter Pylori and Treatments $\$ Side Effects: A Double-Blind Placebo-Controlled Randomized Clinical Trial
}

Nooshin Naghibzadeh

Birjand University of Medical Sciences

Fatemeh Salmani

Birjand University of Medical Sciences

Samira Nomiri

Birjand University of Medical Sciences

Tahmine Tavakoli ( $\nabla$ tahminetavakoli95238@yahoo.com )

Birjand University of Medical Sciences

Research Article

Keywords: Eradication Rate of H. Pylori, Quadruple Therapy, Saccharomyces Boulardii, Lactobacillus Reuteri (DSMZ 17648), Treatments $₫$ Side Effects

Posted Date: October 18th, 2021

DOl: https://doi.org/10.21203/rs.3.rs-910711/v1

License: (c) (i) This work is licensed under a Creative Commons Attribution 4.0 International License.

Read Full License

Version of Record: A version of this preprint was published at BMC Gastroenterology on March 7th, 2022. See the published version at https://doi.org/10.1186/s12876-022-02187-z. 


\section{Abstract}

Background: The primary goal of this placebo-controlled study was to determine the effect of quadruple treatment with Saccharomyces boulardii or Lactobacillus reuteri on the eradication of Helicobacter pylori and side effects of the treatment.

Results: This study was a double-blind, randomized, placebo-controlled trial. And, eradication of helicobacter pylori was reported comparing conventional anti-H. Pylori therapy versus conventional therapy supplemented with S. boulardii and L. reuteri DSMZ 17648. For this aim, a total of 156 patients were included in the current study; and patients positive for $\mathrm{H}$. Pylori infection $(n=156)$ were randomly assigned to 3 groups: 52 patients (Group P) received conventional quadruple therapy plus L. reuteri, 52 patients (Group S) received conventional quadruple therapy plus S. boulardii daily, for 2 weeks, and 52 patients were in the control group (Group C). At the end of the treatment period, all the subjects continued to take proton pump inhibitor (PPI) alone for 14 days, and then, no medication was given for 2 weeks again. During follow-up, gastrointestinal symptoms were assessed using an evaluation scale (Glasgow dyspepsia questionnaire (GDQ)), and adverse events were assessed at 7, 14, 21, and 28 days. As a result, eradication therapy was effective for $94.2 \%$ of subjects in Group S, $92.3 \%$ of subjects in Group P, and $86.5 \%$ of subjects in the control group, with no differences between treatment arms. In Group $S$, the chance of developing symptoms of nausea $(O R=2.74)$, diarrhea $(O R=3.01)$, headache $(O R=10.51)$, abdominal pain $(\mathrm{OR}=3.21)$, and anxiety $(\mathrm{OR}=3.58)$ was significantly lower than in the control group $(\mathrm{p}<0.05)$.

Conclusion: In general, our findings revealed that the use of probiotic supplements containing S. boulardii or Lactobacillus reuteri (DSMZ 17648 strain) improved the eradication rate of $\mathrm{H}$. Pylori infection, but it was not statistically significant. Therefore, it is recommended to conduct future research with a larger sample size to investigate the effect of $\mathrm{S}$. boulardii supplementation on eradicating $\mathrm{H}$. pylori infection and reducing treatment side effects.

Trial registration: IRCT20200106046021N1, this trial was registered on Jan 14, 2020.

\section{Introduction}

Helicobacter pylori is a gram-negative infective bacterium affecting more than half of the world's population [1]. H. Pylori infection contributes to etiology of a variety of diseases, such as gastric ulcer disease, dyspepsia, lymphoma, and gastric cancer [2-4]. Treatment of H. Pylori infection is still being debated around the world, owing to emergence of multidrug-resistant strains of H. Pylori. At the present, the most effective treatment options for $\mathrm{H}$. Pylori infection are complete pathogen eradication using multiple combinations of proton pump inhibitors (PPI) and two or three antibiotics [5-7]. This complicated approach carries a high risk of side effects, antibiotic resistance, and incompatibility $[8,9]$. Reduced $\mathrm{H}$. Pylori load in the stomach via selective bacterial-bacterial surface interaction suggests an alternative therapeutic approach to prevention of the risk posed by this human pathogen. Bacterial 
accumulation has previously been discussed in terms of infection elimination via specific binding to pathogens and formation of co-aggregates [10-12]. Generally, probiotics have potentially beneficial effects in treatment and prevention of various gastrointestinal diseases [13]. These living organisms or their metabolites are administered either alone or in combination with conventional antibiotics. They can regulate normal humans $₫$ flora and promote health by preventing antibiotics $₫$ side effects, increasing elimination rate, stimulating immune response, producing inhibitors, inhibiting toxins, and competing for binding sites and also, they have nutrients and directly compete with pathogenic bacteria [14, 15].Furthermore, changes in gastrointestinal permeability are a precursor to development of injuries like ulcers. Probiotics can help to maintain intestinal barrier by increasing secretion of gastric mucin, decreasing bacterial overgrowth, stimulating local immune responses, and releasing antioxidants [16]. Many observations support the use of probiotics with bioactive components in people infected with $\mathrm{H}$. Pylori, recommending the use of multiplex probiotic microorganisms in control and treatment of infections caused by $\mathrm{H}$. Pylori $[16,17]$.The most common probiotics used in clinical trials are saccharomyces boulardii and Lactobacillus reuteri strains. Lactobacillus species are acid resistant and are known as part of transient gastric flora; their concentration in normal humans $\mathbb{\Delta}$ stomach ranges from 0 to $10^{3}$ per milliliter. They can survive for up to 2 hours in the stomach, and some strains can attach to gastric epithelial cells in-vitro, possibly due to lipoic acid [16]. It has been proposed that Lactobacillus supplementation may be effective in accelerating removal of $\mathrm{H}$. Pylori in first-time patients, as well as having a positive effect on some side effects associated with $\mathrm{H}$. Pylori treatment [18]. To date, saccharomyces boulardii and several lactobacillus reuteri strains have been used alone or in combination with various $\mathrm{H}$. Pylori treatment regimens in clinical studies [19-32]. Therefore, purpose of this study was investigating whether adding saccharomyces boulardii (DAILY EAST ${ }^{\circledR}$ ) probiotic or a supplement of DSMZ 17648 Lactobacillus reuteri (PYLOSHOT ${ }^{\circledR}$ ) to the standard quadruple treatment for $\mathrm{H}$. Pylori infection after 14 days increases eradication rate and reduces the side effects. Due to lack of sufficient studies in this field, there is a need for a clinical trial and it is hoped that the results of this study will be useful in improving treatment of $\mathrm{H}$. pylori.

\section{Method And Materials}

A total of 156 patients were included in the current study; and patients positive for H. Pylori infection (n =156) were randomly assigned to 3 groups: 52 patients (Group P) received conventional quadruple therapy plus L. reuteri, 52 patients (Group S) received conventional quadruple therapy plus S. boulardii daily, for 2 weeks, and 52 patients were in the control group (Group C). At the end of the treatment period, all the subjects continued to take proton pump inhibitor (PPI) alone for 14 days, and then, no medication was given for 2 weeks again. During follow-up, gastrointestinal symptoms were assessed using an evaluation scale (Glasgow dyspepsia questionnaire (GDQ)), and adverse events were assessed at 7, 14, 21, and 28 days. The work flow for overall procedure is shown in Fig. 1.

\subsection{Study Design}


This double-blind, randomized placebo-controlled trial was done to evaluate the effect of adding S. boulardii or DSMZ 17648 strain of Lactobacillus reuteri to traditional quadruple therapy on eradication rates, as well as clinical and pathological aspects of $\mathrm{H}$. Pylori infection which was designed according to the Consolidated Standards of Reporting Trials (CONSORT) guideline [33]. Patients who were referred to the Gastroenterology Clinic of the Valiasr Hospital and met all the inclusion criteria were included in the study. Then, patients were randomly assigned to treatment groups using oblique coins.

\subsection{Inclusion Criteria}

Symptomatic $\mathrm{H}$. pylori-positive patients of both sexes aged between 16 - 74 years old whose infection was confirmed by endoscopy and pathology were included in the study.

\subsection{Exclusion Criteria}

Patients with less than 15 years of age, pregnant or lactating women, patients with hepatic, cardiorespiratory, renal, and neoplastic diseases, those receiving antibiotics, PPIs, bismuth salts, or probiotics within the previous 4 weeks, patients with the history of gastric surgery and sensitivity to any of the drugs used in this study, those receiving any medication interfering with action of the lactobacilli, patients with the history of undergoing surgical procedures influencing stomach or small intestine with potential interference with the study, e.g., gastrectomy or gastric bypass, patients with autoimmune disease, organ transplantation, weight changes $>3 \mathrm{~kg}$ over the last 3 months, those with the history of eradication of $\mathrm{H}$. pylori infection, lactose intolerance, oral intake of antibiotics $<3$ months ago, alcohol or drug abuse, and those participating at other clinical trials at the same time were excluded from the study.

\subsection{Ethical Considerations}

This study was approved by the Research Council and Ethics Committee of the Birjand University of Medical Sciences with the ethics code of IR.BUMS.REC.1398.305. It was also registered on the website of the Iranian registry of clinical trials (IRCT) with the following number: IRCT20200106046021N1 on 14/01/2020. An informed written consent was obtained from all the patients. No costs were imposed on patients. The drugs used in the study, as well as the dose administered to patients, had no side effects or toxicity. The final report and analysis were performed without names of the participants in the study. The modified Glasgow dyspepsia questionnaire (GDQ) was used to record dyspeptic symptoms before starting therapy and at the end of the follow-up period.

\subsection{Treatment Protocol}

The interventions were based on the standard quadruple therapy regimen containing bismuth for $\mathrm{H}$. Pylori, which included $1 \mathrm{~g}$ of Amoxicillin twice daily, $500 \mathrm{mg}$ of Clarithromycin twice daily, $40 \mathrm{mg}$ of PPI twice daily, and $120 \mathrm{mg}$ of bismuth subcitrate (two tablets, twice daily). Patients in Group S were given 1 capsule of DAILYEAST ${ }^{\circledR}$ (saccharomyces boulardii supplement $250 \mathrm{mg}$, Zist Takhmir, Tehran, Iran) b.i.d in combination with anti H. pylori quadruple therapy. Patients in Group P were given 2 capsules of PHYLOSHOT ${ }^{\circledR}$ (100 mg of non-viable Lactobacillus reuteri DSMZ 17648, Lactobacillus acidophilus, Lactobacillus casei, and Bifidobacterium lactis $>10^{9} \mathrm{CFU}$, Zist Takhmir, Tehran, Iran) b.i.d in combination 
with anti $\mathrm{H}$. pylori quadruple therapy. Patients in the control group were also given a placebo capsule b.i.d along with anti $\mathrm{H}$. pylori quadruple therapy. Duration of treatment was equal to 14 days.

At the end of the treatment period, all the subjects continued to take PPI alone for 14 days. Then, the patients were given no medication for 2 weeks before having their first fecal antigen test at the end of the second week. Then, after another 4 weeks, they had a second stool antigen test.

\subsection{Evaluation of Treatments $\$ Side Effects and Tolerability}

Using a previously reported questionnaire, the side effect profile and tolerability were assessed during the follow-up period. Subjects were carefully instructed and trained on filling out questionnaires in order to achieve the highest level of compliance in registering any potential treatment-related side effects. Subjects were asked to report any side effect during and thereafter therapy, such as bitter taste, nausea, vomiting, epigastric discomfort, abdominal pain, and diarrhea.

\subsection{Statistical Analysis}

After entering the SPSS 24 software, the data were described using central and dispersion indices for quantitative variables and frequency and agreement tables for qualitative variables. Homogeneity of groups was assessed using analysis of variance (ANOVA) and Chi-Square tests. Marginal logistic model with generalized estimating equation (GEE) approach was used for modeling the changes in complications during the study. In the tests, significance level was set at 0.05 .

\section{Results}

\subsection{Patients}

A total of 156 subjects with confirmed H. Pylori infection, which $60.9 \%$ of them were female and $39.1 \%$ of them were male, with a mean age of $47.76 \pm 13.92$ years old (age range of $16-74$ years old) participated in the study. They were randomly assigned into three study arms: 52 patients were assigned into the $L$. Reuteri group (Group P), 52 patients were assigned into the S. boulardii group (Group S), and 52 patients were assigned into the placebo group (Group $C$ ). The majority of our patients (93.6\%) were from rural areas, housewife (46.8\%), and non-smokers (76.9\%). Dyspepsia was the most common reason for undergoing an endoscopy procedure (64.7\%). At the time of the first endoscopy, the majority of patients had antral gastritis. Antral and corpus predominant gastritis were found in 88.5 and $27.6 \%$ of patients, respectively. The three treatment groups were similar in terms of their demographic, clinical, and endoscopic characteristics at baseline (Table 1) ( $p>0.05)$. 
Table 1

Characteristics of the All groups.

\begin{tabular}{|c|c|c|c|c|}
\hline & Group C & Group S & Group P & $p$-Value \\
\hline \multicolumn{5}{|l|}{ Age (years) } \\
\hline Mean \pm SD & $46.57 \pm 14.20$ & $49.32 \pm 13.42$ & $47.38 \pm 14.25$ & 0.58 \\
\hline \multicolumn{5}{|l|}{ Sex } \\
\hline Male & $34.6 \%(18)$ & $48.1 \%(25)$ & $34.6 \%(18)$ & \multirow[t]{2}{*}{0.26} \\
\hline Female & $65.4 \%(34)$ & $51.9 \%(27)$ & $65.4 \%(34)$ & \\
\hline \multicolumn{5}{|l|}{ Smoking } \\
\hline Yes & $17.3 \%(9)$ & $32.7 \%(17)$ & $19.2 \%(10)$ & \multirow[t]{2}{*}{0.12} \\
\hline No & $82.7 \%(43)$ & $67.3 \%(35)$ & $80.8 \%(42)$ & \\
\hline \multicolumn{5}{|c|}{ Endoscopic reasons } \\
\hline dyspepsia & $59.6 \%(31)$ & $67.3 \%(35)$ & $67.3 \%(35)$ & 0.63 \\
\hline Heart Burn & $25 \%(13)$ & $25 \%(13)$ & $21.2 \%(11)$ & 0.86 \\
\hline \multicolumn{5}{|c|}{ Endoscopic pattern of gastritis } \\
\hline Antral gastritis & $92.3 \%(48)$ & $82.7 \%(43)$ & $90.4 \%(47)$ & 0.26 \\
\hline Corpus gastritis & $23.1 \%(12)$ & $21.2 \%(11)$ & $38.5 \%(20)$ & 0.09 \\
\hline Pan-gastritis & $3.8 \%(2)$ & $11.5 \%(6)$ & $3.8 \%(2)$ & 0.22 \\
\hline Duodenal ulcer & $3.8 \%(2)$ & $0.0 \%(0)$ & $1.9 \%(1)$ & 0.77 \\
\hline Gastric ulcer & $0.0 \%(0)$ & $1.9 \%(1)$ & $5.8 \%(3)$ & 0.32 \\
\hline Esophagitis & $0.0 \%(0)$ & $0.0 \%(0)$ & $0.0 \%(0)$ & - \\
\hline Normal & $1.9 \%(1)$ & $1.9 \%(1)$ & $0.0 \%(0)$ & 1.00 \\
\hline
\end{tabular}

\subsection{Eradication Rate}

The highest rate of $\mathrm{H}$. pylori eradication occurred in all groups in the second week after treatment. Totally, $86.5,94.2$, and $92.3 \%$ of eradication was observed in the control, $S$, and P groups, respectively. The highest eradication rate was observed in Group S followed by Group P. In general, eradication rate in the studied groups was not statistically significant in the second and sixth weeks, (with $P=0.46$ in the second week and $P=0.53$ in the sixth week, respectively) (Fig. 2).

\subsection{Patients $\$ Compliance and Side Effects}


Headache, abdominal pain, and anxiety were significantly reduced in the Group $S$ (who received saccharomyces boulardii probiotic-DAILYEAST ${ }^{\circledR}$ ). But in Group P, headache and abdominal pain were significantly reduced and anxiety was significantly increased $(p<0.05)$. However, none of the study groups experienced significant changes in vomiting, insomnia, bitter taste in the mouth, or epigastric discomfort ( $p>0.05$ ) (Table 2). According to Table 1, after controlling the effect of time, in the Group S, chance of developing symptoms of headache $(\mathrm{OR}=10.51)$, abdominal pain $(\mathrm{OR}=3.21)$, and anxiety $(\mathrm{OR}=3.58)$ was significantly lower than control group $(p<0.05)$. Also, except for headache $(O R=3.75)$, the Group P did not differ significantly from the control group in incidence of complications ( $p>0.05$ ) (Fig. 3).

Table 2

Generalized Estimating Equations (GEE) logistic Model of the Association between the treatment group and Complications.

\begin{tabular}{|c|c|c|c|c|c|c|}
\hline Outcome & Variables & & Coefficient & SE & P-value & OR \\
\hline \multirow[t]{3}{*}{ Nausea } & Treatment group & $S$ & 0.90 & 0.36 & 0.01 & 2.47 \\
\hline & Base line: Control group & $P$ & 0.41 & 0.36 & 0.26 & 1.52 \\
\hline & time & & 1.51 & 0.14 & $0.001>$ & 4.55 \\
\hline \multirow[t]{3}{*}{ Diarrhea } & Treatment group & $S$ & 1.10 & 0.49 & 0.02 & 3.01 \\
\hline & Base line: Control group & $\mathrm{P}$ & 0.63 & 0.51 & 0.21 & 1.89 \\
\hline & time & & 1.74 & 0.16 & $0.001>$ & 5.74 \\
\hline \multirow[t]{3}{*}{ Headache } & Treatment group & $S$ & 2.35 & 0.65 & $0.001>$ & 10.51 \\
\hline & Base line: Control group & $\mathrm{P}$ & 1.32 & 0.55 & 0.01 & 3.75 \\
\hline & time & & 1.08 & 0.26 & $0.001>$ & 2.95 \\
\hline \multirow[t]{3}{*}{ Abdominal pain } & Treatment group & $S$ & 1.16 & 0.54 & 0.03 & 3.21 \\
\hline & Base line: Control group & $P$ & 0.39 & 0.43 & 0.36 & 1.48 \\
\hline & time & & 1.14 & 0.17 & $0.001>$ & 3.14 \\
\hline \multirow[t]{3}{*}{ Anxiety } & Treatment group & $S$ & 1.27 & 0.56 & 0.02 & 3.58 \\
\hline & Base line: Control group & $\mathrm{P}$ & -0.38 & 0.46 & 0.40 & 0.68 \\
\hline & time & & 0.61 & 0.12 & $0.001>$ & 1.86 \\
\hline
\end{tabular}

\section{Discussion}

Treatment of $\mathrm{H}$. Pylori infection is becoming increasingly important, particularly in developing countries. Despite availability of various therapeutic regimens, treatment failure has remained a growing problem in daily clinical practices. Several factors could contribute to failure of eradication, but the most important factors are antibiotic resistance and clinical efficacy [34]. According to findings of the current study, 
eradication rate of $\mathrm{H}$. Pylori infection was higher in case groups (those who received probiotics) than the control group (receiving placebo). However, there was no statistically significant difference between the study results at the second and sixth weeks after treatment. These findings were consistent with findings of the study by Pourmasoumi et al., who discovered that adding symbiotic to standard treatment can improve eradication rates of $\mathrm{H}$. Pylori infection [28]. Zojaji et al., in a study on evaluating efficacy and safety of adding S. boulardii to standard triple therapy found that adding this probiotic to standard treatment decreased $\mathrm{H}$. Pylori eradication; however, this finding was not significant, which was consistent with findings of our study [30].Furthermore, Cindoruk et al., in a study on assessing the effect of S. boulardii on eradication of $\mathrm{H}$. Pylori infection and reduction of side effects reported no significant difference in $\mathrm{H}$. Pylori eradication between the study groups (71\% in the S. boulardii-treated group and $60 \%$ in the placebo group), which was consistent with our findings [35]. Also, Shavakhi et al., found that using a combination of probiotics containing Lactobacillus, Bifidobacterium, and Streptococcus thermophilus species along with a standard quadruple therapy had no beneficial effect in treating $\mathrm{H}$. Pylori infection. This could be due to the probiotic diet's low dose or high frequency of upper gastrointestinal $\mathbb{Z}$ side effects, which can reduce $\mathrm{H}$. Pylori eradication [36]. Poonyam et al., indicated that eradication of $\mathrm{H}$. Pylori was significantly increased compared to the control group when Lactobacillus reuteri was used in combination with a standard quadruple therapy, which was not in agreement with our finding [37]. Among the reasons for this mismatch, one can mention a difference in type of probiotic used in the two studies in terms of the strains used. Yu et al., demonstrated that Lactobacillus can significantly eradicate $\mathrm{H}$. Pylori in a meta-analysis with a sample size of 724 patients to investigate the probiotic effect of Lactobacillus in combination with a triple eradication regimen [38], which was in contrary to our findings. Zhou et al., in a meta-analysis showed that $\mathrm{S}$. boulardii can significantly increase eradication of $\mathrm{H}$. Pylori [39], which was not consistent with our findings. The large sample size and multi-center nature of these studies are two reasons for inconsistency between the findings. According to findings of our study, distribution frequency of some side effects in the subjects receiving S. boulardii probiotic was significantly reduced after the intervention but, except for headache, no significant change was observed in the Lactobacillus reuteri-treated group. According to the findings, the most common side effects in the first week were vomiting, bitter taste in the mouth, epigastric discomfort, and insomnia, which were decreased after that time. However, no statistically significant difference was found between the studied groups at different times. Our findings were consistent with those of the studies by Pourmasoumi et al., and Lv et al., who showed that probiotic administration can reduce the side effects of anti H. pylori treatment [29, 31]. Zojaji et al., discovered that using S. boulardii supplement significantly reduces side effects, such as nausea, diarrhea, epigastric discomfort, and bloating in the first and second weeks of treatment [30], which was in agreement with the findings of our study on reduction of side effects after taking S. boulardii supplement. Furthermore, Cindoruk et al., in a study found that the side effects of epigastric discomfort and dyspepsia were significantly reduced in the group that received S. boulardii [35]. Shavakhi et al., reported that incidence of diarrhea was significantly lower in the group that consumed combined probiotics containing Lactobacillus, Bifidobacterium, and Streptococcus thermophilus than the placebo group. On the other hand, incidence of abdominal pain was significantly higher in the probiotic group than the placebo group [36]. Frequency of nausea, vomiting, epigastric 
discomfort, and bitter taste was significantly reduced in the group that received oral supplement of Lactobacillus reuteri compared to the placebo group in the study by Poonyam et al., which was not in line with the results of our study [37]. Yu et al.,[38] in a meta-analysis found that Lactobacillus reduces treatment-related side effects, which was in contrary to our findings. Zhou et al., discovered that $\mathrm{S}$. boulardii could significantly reduce treatments $\$ side effects, particularly diarrhea and constipation [39], which was consistent with our findings.

\section{Limitations Of The Study}

The main limitation of this study was poor cooperation of patients with the researcher to conduct the research process. For eliminating it, patients were assured that the treatment was appropriate for them and the patient's conditions were improved and in case of problems, necessary services were provided free of charge.

\section{Conclusion}

In general, our findings revealed that the use of probiotic supplements containing S. boulardii or Lactobacillus reuteri (DSMZ 17648 strain) improved eradication rate of $\mathrm{H}$. Pylori infection, but it was not statistically significant. On the other hand, $\mathrm{S}$. boulardii could significantly reduce some side effects of $\mathrm{H}$. Pylori eradication therapy. But, effectiveness of Lactobacillus reuteri on these cases was not significant and only headache was remarkably reduced, which was in accordance with the previous evidences in the literature. Therefore, it is recommended to conduct the future research with larger sample size in order to investigate the effect of $\mathrm{S}$. boulardii supplementation on eradicating $\mathrm{H}$. pylori infection and reducing

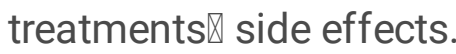

\section{Declarations}

\section{Ethics approval and consent to participate}

All methods were carried out in accordance with relevant guidelines and regulations. This study was approved by the Research Council and Ethics Committee of the Birjand University of Medical Sciences with the ethics code of IR.BUMS.REC.1398.305. It was also registered on the website of the Iranian registry of clinical trials (IRCT) with the following number: IRCT20200106046021N1 on 14/01/2020. Informed written consent was obtained from all the patients. No costs were imposed on patients. The drugs used in the study, as well as the dose administered to patients, had no side effects or toxicity. The final report and analysis were performed without the names of the participants in the study.

\section{Consent for publication}

Not Applicable. 


\section{Availability of data and materials}

The datasets used and analyzed during the current study are available from the corresponding author on reasonable request.

\section{Competing interests}

The authors declare that they have no competing interests.

\section{Funding}

Not applicable.

\section{Author contribution}

N.N. Coordinated the experiments, F.S. performed the Statistical analysis and Interpretated the data, S.N. Prepared the manuscript, T.T Revised the important intellectual content and Supervision, all authors reviewed the manuscript.

\section{Acknowledgements}

Not applicable.

\section{References}

1. Hooi JK, Lai WY, Ng WK, Suen MM, Underwood FE, Tanyingoh D, Malfertheiner P, Graham DY, Wong VW, Wu JC: Global prevalence of Helicobacter pylori infection: systematic review and meta-analysis. Gastroenterology 2017, 153(2):420-429.

2. Sjomina $\mathrm{O}$, Heluwaert $F$, Moussata $D$, Leja M: Helicobacter pylori infection and nonmalignant diseases. Helicobacter 2017, 22:e12408.

3. Uemura N, Okamoto S, Yamamoto S, Matsumura N, Yamaguchi S, Yamakido M, Taniyama K, Sasaki $\mathrm{N}$, Schlemper RJ: Helicobacter pylori infection and the development of gastric cancer. N Engl J Med 2001, 345(11):784-789.

4. Wotherspoon AC, Ortiz-Hidalgo C, Falzon MR, Isaacson PG: Helicobacter pylori-associated gastritis and primary B-cell gastric lymphoma. The Lancet 1991, 338(8776):1175-1176.

5. Nishizawa T, Suzuki H: Mechanisms of Helicobacter pylori antibiotic resistance and molecular testing. Front Mol Biosci 2014, 1:19. 
6. Fiorini G, Zullo A, Vakil N, Saracino IM, Ricci C, Castelli V, Gatta L, Vaira D: Rifabutin triple therapy is effective in patients with multidrug-resistant strains of Helicobacter pylori. J Clin Gastroentero/ 2018, 52(2):137-140.

7. Thung I, Aramin H, Vavinskaya V, Gupta S, Park J, Crowe S, Valasek M: the global emergence of Helicobacter pylori antibiotic resistance. Aliment Pharmacol Ther 2016, 43(4):514-533.

8. Zhang M: High antibiotic resistance rate: A difficult issue for Helicobacter pylori eradication treatment. World J Gastroenterol2015, 21(48):13432.

9. Malfertheiner P, Megraud F, O'morain C, Gisbert J, Kuipers E, Axon A, Bazzoli F, Gasbarrini A, Atherton J, Graham DY: Management of Helicobacter pylori infection-the Maastricht V/Florence consensus report. Gut 2017, 66(1):6-30.

10. Younes JA, van der Mei HC, van den Heuvel E, Busscher HJ, Reid G: Adhesion forces and coaggregation between vaginal staphylococci and lactobacilli. PloS one 2012, 7(5):e36917.

11. Lang C, Böttner M, Holz C, Veen M, Ryser M, Reindl A, Pompejus M, Tanzer J: Specific lactobacillus/mutans streptococcus co-aggregation. J Dent Res 2010, 89(2):175-179.

12. McMillan A, Dell M, Zellar MP, Cribby S, Martz S, Hong E, Fu J, Abbas A, Dang T, Miller W: Disruption of urogenital biofilms by lactobacilli. Colloids and Surfaces B: Biointerfaces 2011, 86(1):58-64.

13. Ritchie ML, Romanuk TN: A meta-analysis of probiotic efficacy for gastrointestinal diseases. PloS one 2012, 7(4):e34938.

14. Verna EC, Lucak S: Use of probiotics in gastrointestinal disorders: what to recommend? Therap Adv Gastroenterol 2010, 3(5):307-319.

15. Domingo JJS: Review of the role of probiotics in gastrointestinal diseases in adults. Gastroenterología y Hepatología [English Edition] 2017, 40(6):417-429.

16. Gotteland $\mathrm{M}$, Brunser $\mathrm{O}$, Cruchet $\mathrm{S}$ : Systematic review: are probiotics useful in controlling gastric colonization by Helicobacter pylori? Aliment Pharmacol Ther 2006, 23(8):1077-1086.

17. Vítor JM, Vale FF: Alternative therapies for Helicobacter pylori: probiotics and phytomedicine. FEMS Immunol Med Microbio/2011, 63(2):153-164.

18. Zou J, Dong J, Yu X: Meta-analysis: Lactobacillus containing quadruple therapy versus standard triple first-line therapy for Helicobacter pylori eradication. Helicobacter 2009, 14(5):449-459.

19. Francavilla R, Lionetti E, Castellaneta SP, Magistà AM, Maurogiovanni G, Bucci N, De Canio A, Indrio F, Cavallo L, lerardi E: Inhibition of Helicobacter pylori infection in humans by Lactobacillus reuteri ATCC 55730 and effect on eradication therapy: a pilot study. Helicobacter 2008, 13(2):127-134.

20. Dore MP, Cuccu M, Pes GM, Manca A, Graham DY: Lactobacillus reuteri in the treatment of Helicobacter pylori infection. Intern Emerg Med 2014, 9(6):649-654.

21. Emara MH, Mohamed SY, Abdel-Aziz HR: Lactobacillus reuteri in management of Helicobacter pylori infection in dyspeptic patients: a double-blind placebo-controlled randomized clinical trial. Therap Adv Gastroenterol 2014, 7(1):4-13. 
22. Busjahn A, Lang C: Significant reduction in Helicobacter pylori load in humans with Lactobacillus reuteri DSMZ 17648. 2010.

23. Mehling $H$, Busjahn A: Non-viable Lactobacillus reuteri DSMZ 17648 (Pylopass ${ }^{\mathrm{TM}}$ ) as a new approach to Helicobacter pylori control in humans. Nutrients 2013, 5(8):3062-3073.

24. Holz C, Busjahn A, Mehling H, Arya S, Boettner M, Habibi H, Lang C: Significant reduction in Helicobacter pylori load in humans with non-viable Lactobacillus reuteri DSM17648: a pilot study. Probiotics Antimicrob 2015, 7(2):91-100.

25. Saulnier DM, Santos F, Roos S, Mistretta T-A, Spinler JK, Molenaar D, Teusink B, Versalovic J: Exploring metabolic pathway reconstruction and genome-wide expression profiling in Lactobacillus reuteri to define functional probiotic features. PloS one 2011, 6(4):e18783.

26. Yadegar A, Mobarez AM, Alebouyeh M, Mirzaei T, Kwok T, Zali MR: Clinical relevance of cagL gene and virulence genotypes with disease outcomes in a Helicobacter pylori infected population from Iran. World J Microbiol Biotechnol 2014, 30(9):2481-2490.

27. Yadegar A, Alebouyeh M, Zali MR: Analysis of the intactness of Helicobacter pylori cag pathogenicity island in Iranian strains by a new PCR-based strategy and its relationship with virulence genotypes and EPIYA motifs. Infect Genet Evol 2015, 35:19-26.

28. Pourmasoumi M, Najafgholizadeh A, Hadi A, Mansour-Ghanaei F, Joukar F: The effect of synbiotics in improving Helicobacter pylori eradication: A systematic review and meta-analysis. Complement Ther Med 2019, 43:36-43.

29. Lv Z, Wang B, Zhou X, Wang F, Xie Y, Zheng H, Lv N: Efficacy and safety of probiotics as adjuvant agents for Helicobacter pylori infection: A meta-analysis. Exp Ther Med 2015, 9(3):707-716.

30. Zojaji H, Ghobakhlou M, Rajabalinia H, Ataei E, Sherafat SJ, Moghimi-Dehkordi B, Bahreiny R: The efficacy and safety of adding the probiotic Saccharomyces boulardii to standard triple therapy for eradication of Helicobacter pylori; a randomized controlled trial. Gastroenterol Hepatol Bed Bench 2013, 6:Hepatol Bed Bench 2013; 2016 (Suppl. 2011): S2099-S2104.

31. Dore MP, Bibbo S, Pes GM, Francavilla R, Graham DY: Role of probiotics in Helicobacter pylori eradication: Lessons from a study of Lactobacillus reuteri strains DSM 17938 and ATCC PTA 6475 (Gastrus ${ }^{\circledR}$ ) and a proton-pump inhibitor. Can J Infect Dis 2019, 2019.

32. Buckley M, Lacey S, Doolan A, Goodbody E, Seamans K: The effect of Lactobacillus reuteri supplementation in Helicobacter pylori infection: a placebo-controlled, single-blind study. $B M C$ nutrition 2018, 4(1):1-8.

33. KF S, DG A, and D M: CONSORT 2010 Statement: updated guidelines for reporting parallel group randomised trials. Int J Surg (London, England) 2011, 9(8):672-677.

34. Ibrahim NH: The use of different laboratory methods in diagnosis of Helicobacter pylori infection; a comparative study. 2012.

35. Cindoruk M, Erkan G, Karakan T, Dursun A, Unal S: Efficacy and safety of Saccharomyces boulardii in the 14-day triple anti-Helicobacter pylori therapy: a prospective randomized placebo-controlled double-blind study. Helicobacter 2007, 12(4):309-316. 
36. Shavakhi A, Tabesh E, Yaghoutkar A, Hashemi H, Tabesh F, Khodadoostan M, Minakari M, Shavakhi $S$, Gholamrezaei $A$ : The effects of multistrain probiotic compound on bismuth-containing quadruple therapy for Helicobacter pylori infection: a randomized placebo-controlled triple-blind study. Helicobacter 2013, 18(4):280-284.

37. Poonyam P, Chotivitayatarakorn P, Vilaichone RK: High Effective of 14-Day High-Dose PPI- BismuthContaining Quadruple Therapy with Probiotics Supplement for Helicobacter Pylori Eradication: A Double Blinded-Randomized Placebo-Controlled Study. Asian Pac J Cancer Prev 2019, 20(9):28592864.

38. Yu M, Zhang R, Ni P, Chen S, Duan G: Efficacy of Lactobacillus-supplemented triple therapy for $\mathrm{H}$. pylori eradication: A meta-analysis of randomized controlled trials. PloS one 2019, 14(10):e0223309e0223309.

39. Zhou B-G, Chen L-X, Li B, Wan L-Y, Ai Y-W: Saccharomyces boulardii as an adjuvant therapy for Helicobacter pylori eradication: A systematic review and meta-analysis with trial sequential analysis. Helicobacter 2019, 24(5):e12651.

\section{Figures}

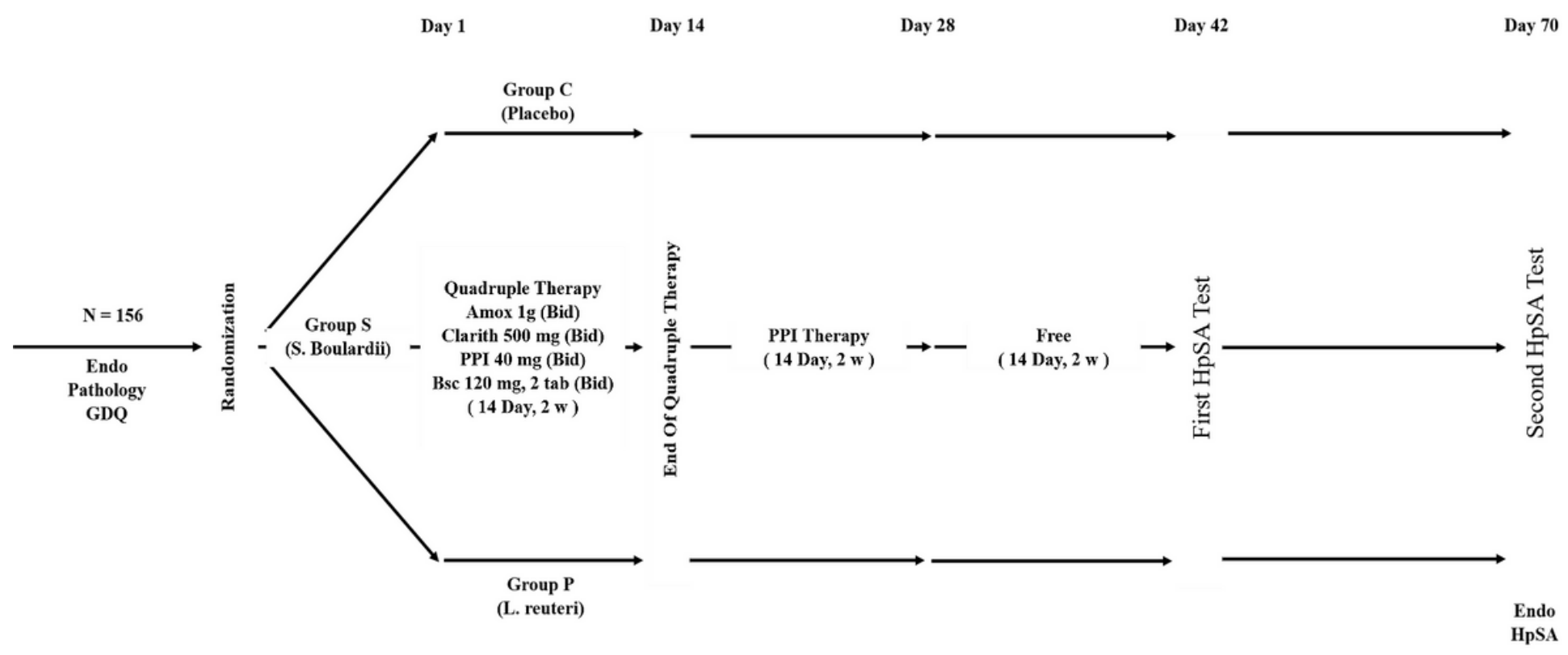

Figure 1

Flow chart of the study. A total of 156 patients met the inclusion criteria. HpSA: H. pylori stool antigen, Endo: endoscopic, GDQ: Glasgow dyspepsia questionnaire, PPI: proton pomp inhibitor, Amox: amoxicillin, Clarith: clarithromycin, b.i.d: twice daily, BSC: bismuth subcitrate 


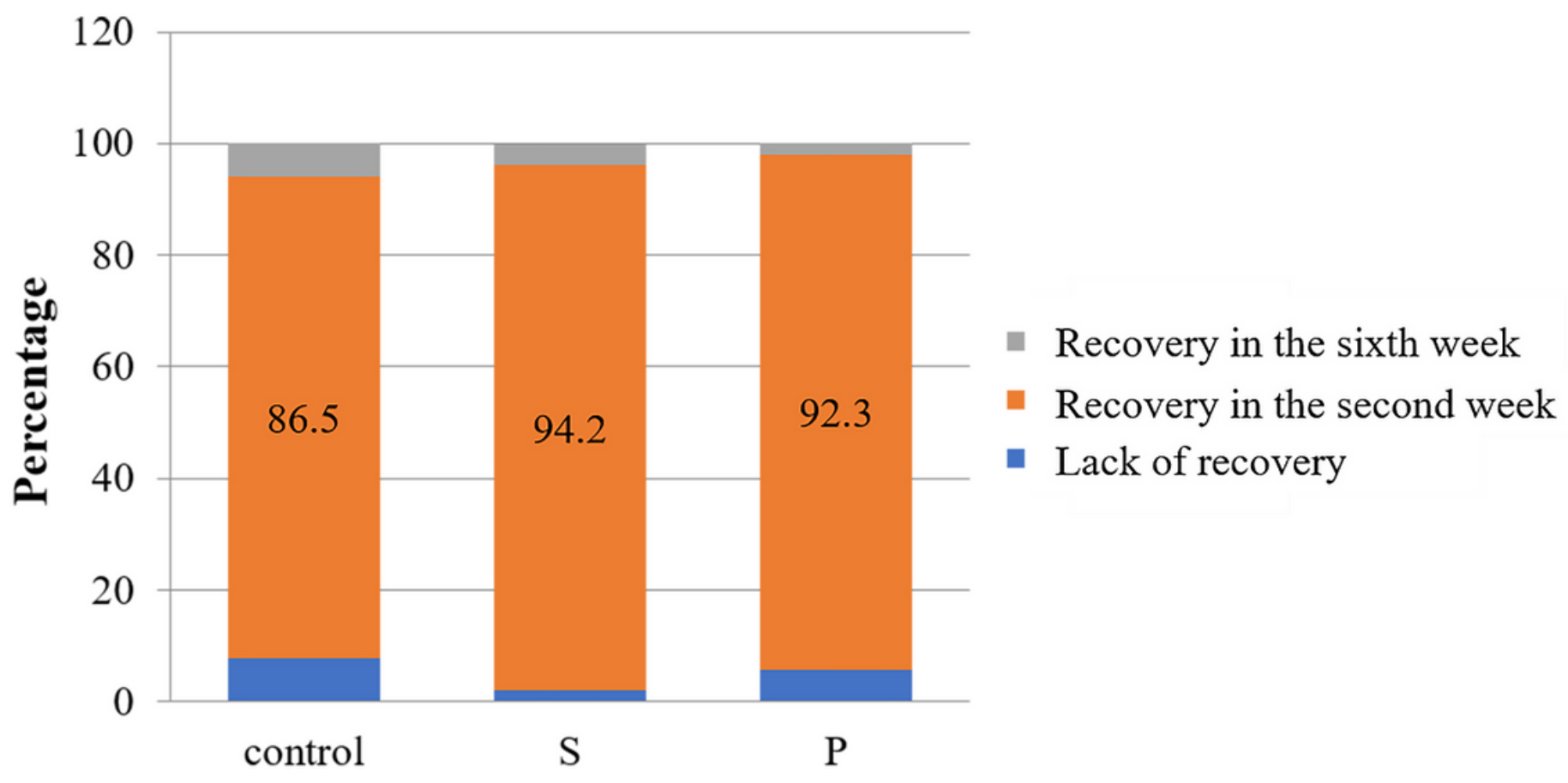

Figure 2

Rate of eradication of $\mathrm{H}$. Pylori infection in the studied groups

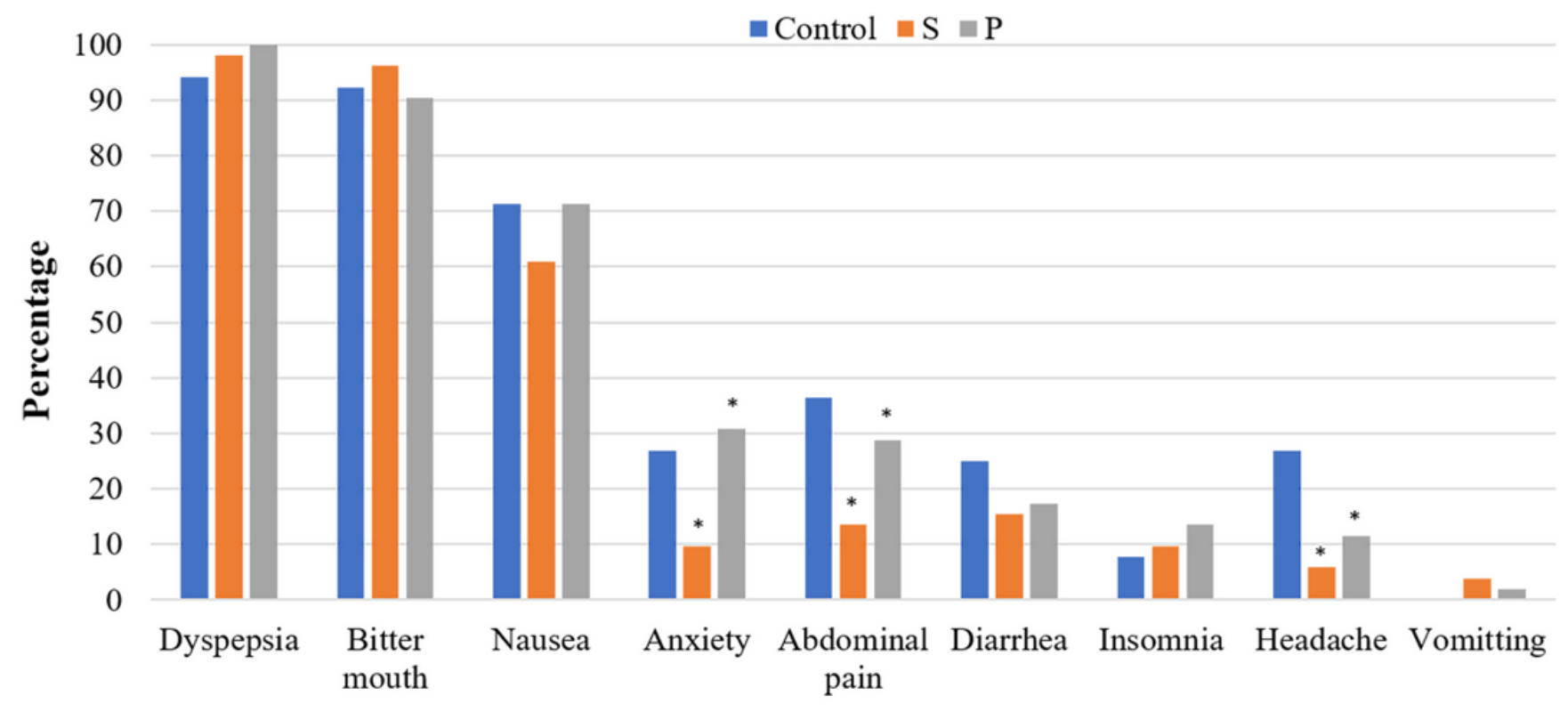

Figure 3

Distribution frequency of side effects observed in the study groups 